\title{
A DRIFT FREE NERNSTIAN IRIDIUM OXIDE PH SENSOR
}

\author{
J. Hendrikse, W. Olthuis and P.Bergveld \\ MESA Research Institute, University of Twente \\ P.O. Box 217, 7500AE Enschede, The Netherlands \\ E-mail: j.hendrikse@el.utwente.nl \\ Fax: x-31 534892287
}

\section{SUMMARY}

A novel way of eliminating drift problems in metal oxide $\mathrm{pH}$ sensors is presented. The method employs a FETstructure under the electrode that uses the metal oxide as a gate contact. In addition to the enhanced drift properties, the new sensor has an almost ideal nernstian response. First a theoretical explanation is given, which is then confirmed by measurements.

Keywords: pH sensor, drift, FET-structure

\section{INTRODUCTION}

Potentiometric metal oxide $\mathrm{pH}$ sensors have been proposed and tested previously [1], but the drift problems that are caused by redox couples present in the solution have not been solved. In this paper we present and successfully test a method for the removal of this type of drift. A second problem is the non-nernstian response of most of these sensors. The $\mathrm{pH}$ dependence of the sensor we present is almost ideal, even though we chose iridium oxide as an electrode material, which is notorious for its non-nernstian behavior.

\section{DRIFT PROBLEMS IN NOBLE METAL OXIDE PH ELECTRODES}

Electrodes made of hydrated metal oxides $\mathrm{MeO}(\mathrm{OH})$, where Me can be Ir,W,Pt, or any other of a number of metals [2], take part in redox reactions of the type $\mathrm{MeO}(\mathrm{OH}) \leftrightarrow \mathrm{MeO}_{2}$ $+\mathrm{H}^{+}+\mathrm{e}$. For the potential of these electrodes with respect to a reference, $\mathrm{E}$, it is easily derived that

$$
E=E^{0}-\frac{k T}{q} \ln \left(\frac{\gamma_{\mathrm{MeO}(\mathrm{OH})}[\mathrm{MeO}(\mathrm{OH})]}{\gamma_{\mathrm{MeO}_{2}}\left[\mathrm{MeO}_{2}\right]}\right)+\frac{k T}{q} \ln \left(a_{H^{+}}\right)(1)
$$

where $\mathrm{k}, \mathrm{T}$ and $\mathrm{q}$ have their usual meaning, $\mathrm{E}^{0}$ is the standard electrode potential and $a_{i}=\gamma_{i}[I]$ is the activity of species $\mathrm{I}$, which for the metal oxide molecules has been split into their activity coefficient $\gamma_{i}$ and concentration [I]. The last term on the right-hand side suggests that the use of these electrodes as nernstian $\mathrm{pH}$ sensors is possible. In their actual use however, two problems arise. When electrochemically active substances, like oxygen, are present in the solution, they influence the concentrations of $\mathrm{MeO}(\mathrm{OH})$ and $\mathrm{MeO}_{2}$. This changes the second term of Eq. (1), thus causing drift. Moreover, the activity coefficients $\gamma_{\mathrm{i}}$ may be $\mathrm{pH}$ dependent, thus causing a non-nernstian $\mathrm{pH}$ response of the electrode [3]. If the change in the activity coefficients is slow after a change in $\mathrm{pH}$, a second type of drift can be identified with this process. In contrast to the first type of drift, this type of drift can take place even if there are no electrochemically active substances present in the solution and does not involve the exchange of protons. Drift having these characteristics was reported by Petrii and Vitins. [4]

\section{RESOLVING DRIFT PROBLEMS BY MEANS OF AN ${ }^{\mathrm{E}}$ MOSFET}

We propose a new device, denoted the ${ }^{\mathrm{E}}$ MOSFET, where the electrode plays the role of the gate contact in a MOSFET-structure. It can be shown theoretically that the threshold voltage of the ${ }^{\mathrm{E}}$ MOSFET, $\mathrm{V}_{\mathrm{T}}$, depends on the electrode potential, $\mathrm{E}$, and on the $\mathrm{pH}$ of the electrolyte.[5] Therefore, $V_{T}$ suffers not only from exactly the same drift problems as $E$ does, but also from the deviation from the nernstian response that is caused by the $\mathrm{pH}$ dependent activity coefficients:

$$
\begin{array}{r}
V_{T}=C+E^{0}-\frac{k T}{q} \ln \left(\frac{\gamma_{\mathrm{MeO}(\mathrm{OH})}[\mathrm{MeO}(\mathrm{OH})]}{\gamma_{\mathrm{MeO}_{2}}\left[\mathrm{MeO}_{2}\right]}\right)+ \\
+\frac{k T}{q} \ln \left(a_{H^{+}}\right)-\Delta \phi_{\mathrm{E}}^{\mathrm{M}}
\end{array}
$$

where $\mathrm{C}$ is a constant and $\Delta \phi_{E}^{M}$ is the potential difference between the metal oxide and the electrolyte solution, which is known to be determined by the $\mathrm{pH}$. If Eq. (2) is subtracted from Eq. (1) we find:

$$
E-V_{T}=-C+\Delta \phi_{E}^{M}
$$

So, what we actually determine when $V_{T}$ is subtracted from $E$ is, apart from a constant factor, the potential difference between the iridium oxide and the solution. In the literature, potentials across the metal oxide - solution interface have been 
4C3.02

studied for oxides of for example $\mathrm{Si}$ and $\mathrm{Al}$. The $\mathrm{pH}$ dependency of these potentials is often lower than the ideal value because the buffer capacity of the oxide surface with regard to protons is not large enough compared to the charge on the double layer capacitance. Tantalum oxide is in this respect an example of nearly ideal behaviour. Since for iridium oxide $\mathrm{E}$ is always measured rather than $\Delta \phi_{E}^{M}$, literature values are unavailable for this oxide.

\section{EXPERIMENTAL}

MOSFET-structures having a gate of $500 \mu \mathrm{m}$ wide and $15 \mu \mathrm{m}$ long were fabricated using standard NMOS processing steps also used in the fabrication of ISFETs. [7] As a gate contact a $\pm 100 \mathrm{~nm}$ thick Sputtered Iridium Oxide Film (SIROF) was deposited on top of the gate oxide by sputtering an Ir target in an $\mathrm{O}_{2} / \mathrm{Ar}$ atmosphere. Iridium oxide films prepared in this way are known to have very small pores resulting in an intimate contact between the film and the electrolyte. The SIROF was shaped as a $2000 \mu \mathrm{m} \times 100 \mu \mathrm{m}$ rectangle symmetrically placed on top of the gate area by the use of lift-off techniques. In order to insulate the relevant parts of the sensor from the solution, a tantalum oxide film was deposited around the gate area, on top of which a polyimide (PI) film was applied to improve the adhesion of the Hysol encapsulation. The resulting structure is shown in Fig. 1. The devices were then mounted on a piece of printed circuit board and packaged using Hysol epoxy resin.

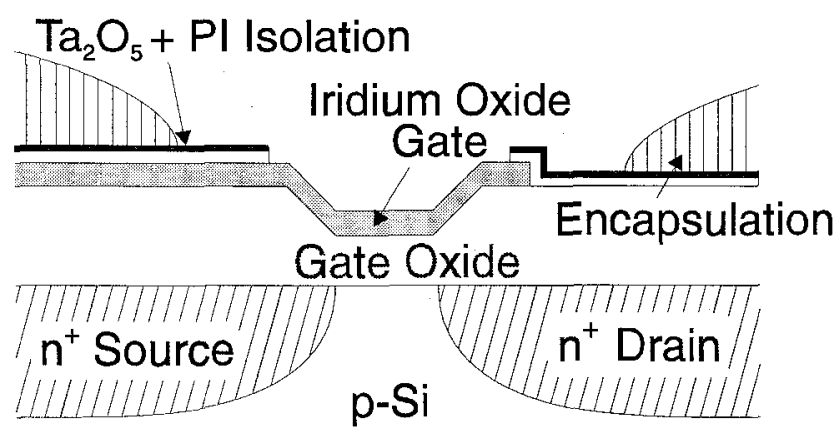

Figure 1: Cross section of the MOSFET-device.
The devices were immersed in an acetic/boric/ortho phosphatic acid buffer $(\mathrm{pH}=2.5)$ at room temperature and both $\mathrm{E}$ and $\mathrm{V}_{\mathrm{T}}$ were determined while the $\mathrm{pH}$ was changed by addition of small quantities of $1.0 \mathrm{M} \mathrm{NaOH}$. In order to maximize the drift of the conventional sensor signal, E, during the experiment, the solution was purged alternately with $\mathrm{N}_{2}$ and air so that the oxygen concentration was varied. In all experiments analytical grade chemicals (Merck) were dissolved in demineralised water having a specific resistance of more than $5 \mathrm{M} \Omega \mathrm{cm}$.

In order to determine $V_{T}$, the FET was connected to a circuit keeping both the drain current and the drain-source voltage at a constant value, adjusting the gate voltage by means of feedback. The output voltage of this circuit tracks any change in the threshold voltage, which is up to a constant identical to the flat band voltage. Since Eq. (3) already involves the unknown constant $\mathrm{C}$, this difference is of no importance. In order to determine $E$, the potential of the SIROF with respect to a Radiometer $\mathrm{REF} 200 \mathrm{Ag} / \mathrm{AgCl}$ reference electrode was measured simultaneously using a voltage follower having a $10^{12} \Omega$ input impedance. The set-up is shown in Fig. 2. Note that the reference electrode is not needed for the $V_{T}$ measurement because the iridium oxide is directly connected to the ground of the amplifier.

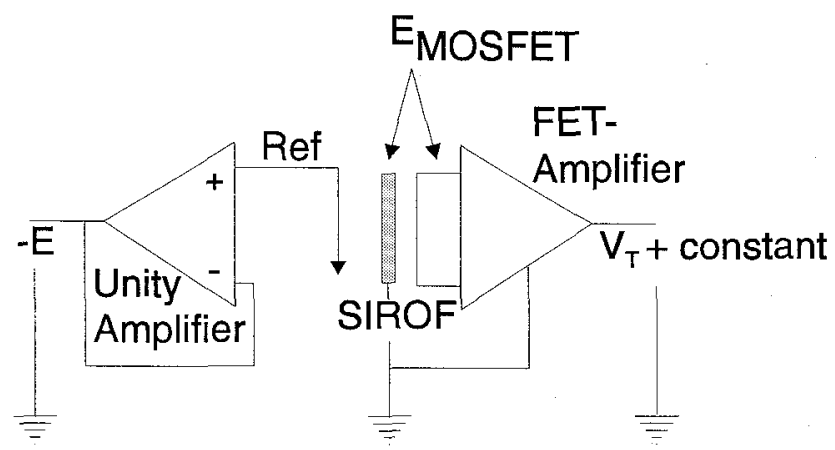

Figure 2: The measurement set-up.

In order to test the drift properties of the new sensor, it was placed in a thermostatted phosphate buffer $(\mathrm{T}=300 \mathrm{~K}$, $\mathrm{pH}=4.85$ ) and both $\mathrm{E}$ and $\mathrm{V}_{\mathrm{T}}$ were monitored for 19 hours using the same set-up. 


\section{RESULTS}

As can be seen in Fig. 3, the alternating purging of the solution with $\mathrm{N}_{2}$ and Air makes both $\mathrm{E}$ and $\mathrm{V}_{\mathrm{T}}$, behave in an inpredictable manner, though a sudden change in $E$ can be noticed at the moments when the $\mathrm{pH}$ is changed.

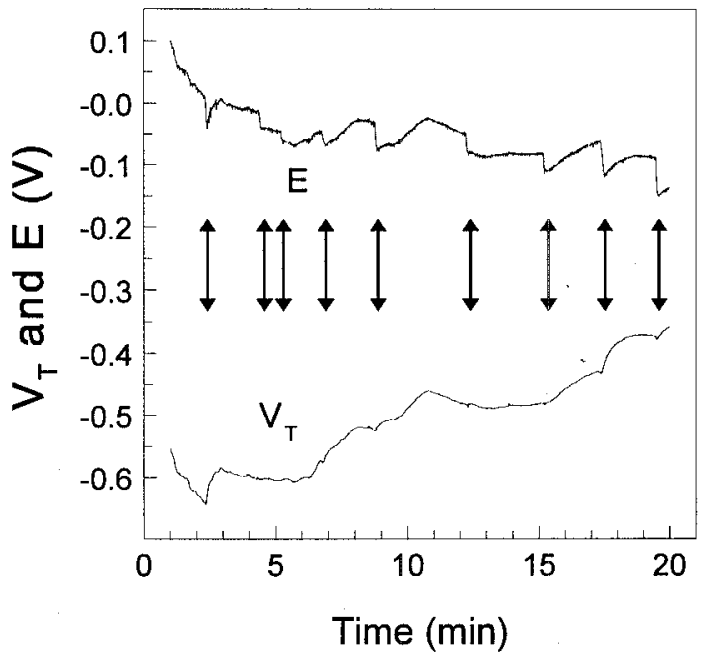

Figure 3: The electrode potential and threshold voltage as a function of time, while alternately purging with $\mathrm{N}_{2}$ and air. At the same time the $\mathrm{pH}$ was changed, as is indicated by the arrows.

The difference between the two, $\mathrm{E}-\mathrm{V}_{\mathrm{T}}$, is presented in Fig. 4, and reacts in a perfectly stepwise fashion to the $\mathrm{pH}$ changes.

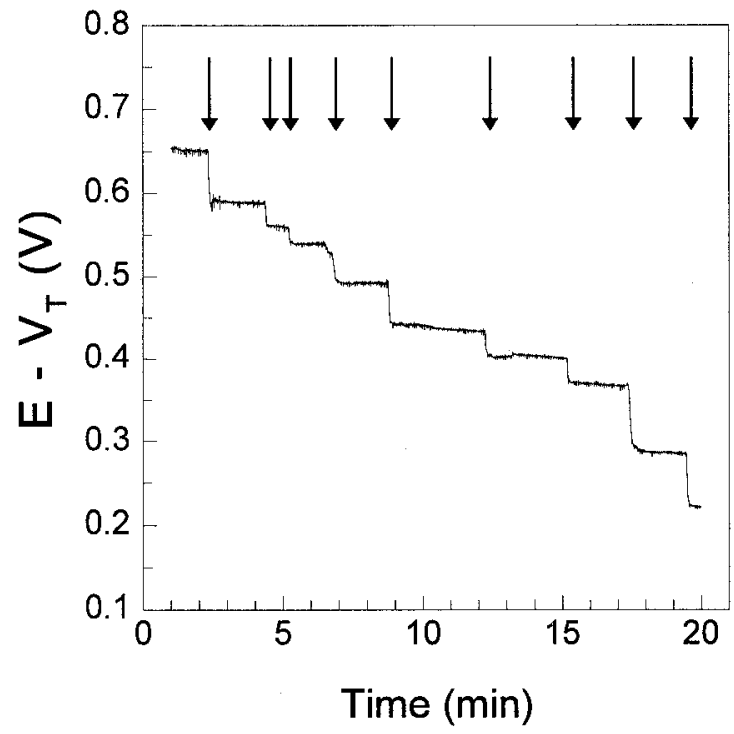

Figure 4: The threshold voltage minus the electrode potential as a function of time. PH changes are indicated by arrows

Moreover, when the height of the steps in Fig. 4 is determined as a function of the $\mathrm{pH}$, the nernstian response shown in Fig. 5 is the result. Apparently, the buffer capacity of the porous iridium oxide is quite large compared to the charge on the double layer capacitance, [6] thus resulting in an ideal nernstian response.

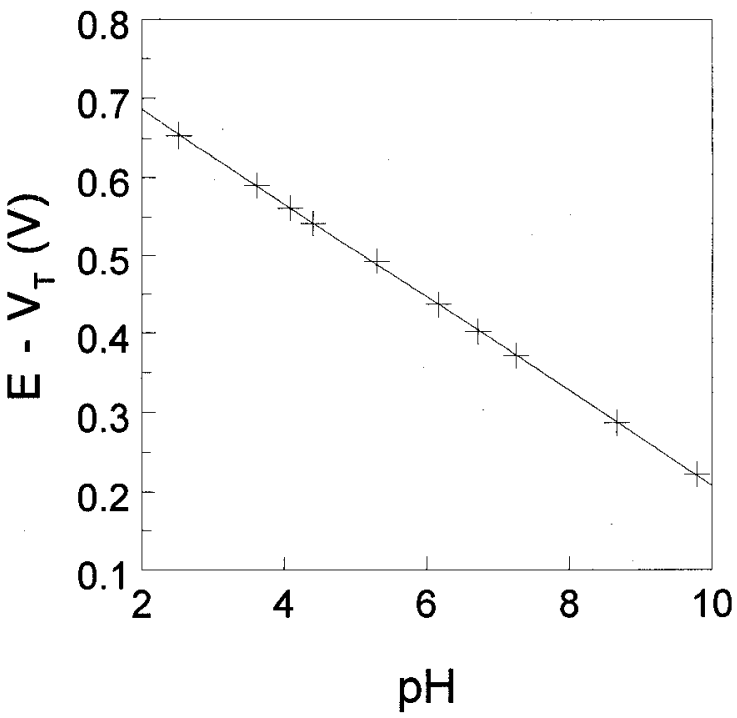

Figure 5: The electrode potential minus the threshold voltage as a function of $\mathrm{pH}$. A linear fit gives a slope of $-59.8 \pm 0.3 \mathrm{mV} / \mathrm{pH}$ with an $r^{2}$ value of 0.99983

The drift properties of the sensor while the solution is continuously in contact with air are shown in Fig. 6. While both $\mathrm{E}$ and $\mathrm{V}_{\mathrm{T}}$ suffer from drift, the sensor signal, $\mathrm{E}-\mathrm{V}_{\mathrm{T}}$, is the same within $13 \mathrm{mV}$ over a period of 19 hours.

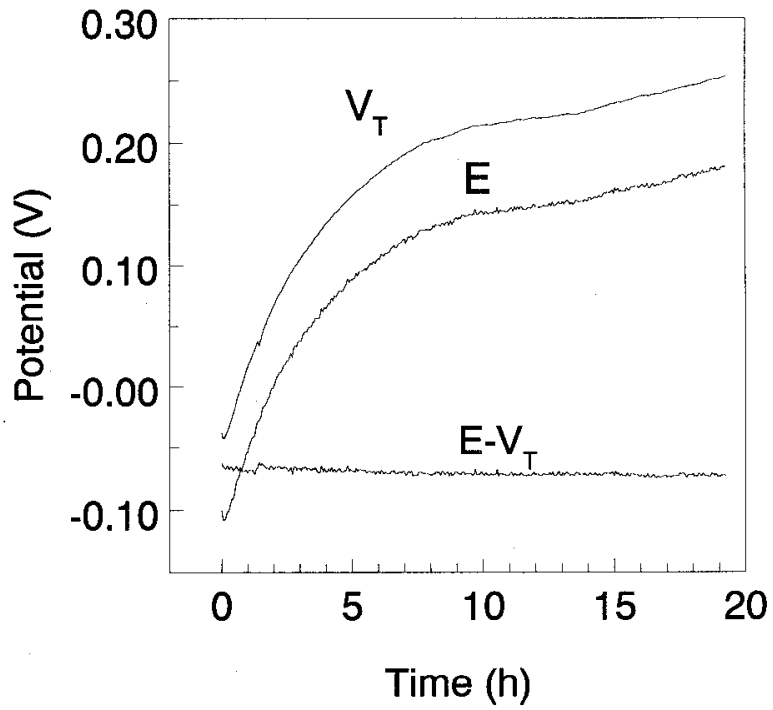

Figure 6: The electrode potential, the threshold voltage and the difference between the two as a function of time. The sensor device was placed in a thermostatted stirred beaker containing a phosphate buffer $(p H=4.85)$. 


\section{DISCUSSION AND CONCLUSION}

As can be seen in Fig. 5, a pH sensor with a nernstian response was developed by subtracting the threshold voltage of a MOSFET having a noble metal oxide gate contact from the usual potentiometric $\mathrm{pH}$ response. Two important problems are thus resolved. Not only is drift arising from oxidizing or reducing agents in the solution eliminated, but also problems due to $\mathrm{pH}$ dependent activity coefficients are solved. The method has a clear theoretical background which is proven by measurements.

As was observed earlier, the nernstian response of the potential across the metal oxide-solution interface that was found means that the buffer capacity of the oxide surface is very large compared to its double layer capacitance. This phenomenon can be explained in the following way. The pores inside the iridium oxide are so small that the formation of an electrical double layer at the outer Helmholz plane is hindered, whereas the adsorption of protons at the inner Helmholz plane is still possible. This means that small pores will be able to enhance the buffer capacity of the iridium oxide while the double layer capacitance is more or less constant.

The drift properties of the new sensor are considerably better than those of classical potentiometric sensors; compare $\mathrm{E}$ and $\mathrm{E}-\mathrm{V}_{\mathrm{T}}$ in Fig. 6. According to the theory, the remaining drift may be caused by changes in the potential across the metal oxide interface or could originate from changes in the "constant" $\mathrm{C}$, possibly due to changes in the FET-structure.

\section{ACKNOWLEDGEMENT}

The authors thank Johan Bomer for technical advice and realization of the devices.

\section{REFERENCES}

[1] A. Fog, R.P. Buck, Sensors and Actuators, 5, 137-146, (1984)

[2] C.G. Granqvist, Handbook of inorganic electrochromic materials, Elsevier 1995

[3] W. Olthuis, M.A.M. Robben, P.Bergveld, M. Bos and W.E. van der Linden, Sensors and Actuators, B4 247-256, 1990

[4] O.A. Petrii and A. Vitinš, Electrokhimiya, 27, 461-476, 1991

[5] J.Hendrikse, W. Olthuis and P. Bergveld, submitted to J. Appl. Phys.

[6] R.E.G. van Hal, J.C.T. Eijkel and P. Bergveld, Adv. Colloid Interface Sci., 31-62, 1996

[7] P. Bergveld and A. Sibbald, Comprehensive Analytical Chemistry Vol. XXIII: Analytical and biomedical applications of ion-selective field-effect transistors, (Elsevier, Amsterdam, 1988) 